\title{
Self-propulsion of bent bimetallic Janus rods
}

\author{
Dugyala Venkateshwar Rao ${ }^{\mathrm{a}}$, Naveen Reddy,c ${ }^{\mathrm{b}, \mathrm{Jan}}$ Fransaer $^{\mathrm{d}}$ and Christian Clasen ${ }^{\mathrm{a} *}$ \\ a Department of Chemical Engineering, KU Leuven, 3001 Leuven, Belgium \\ ${ }^{b}$ Hasselt University, Martelarenlaan 42, 3500 Hasselt, Belgium \\ c IMO-IMOMEC, Wetenschapspark 1, 3590 Diepenbeek, Belgium, Belgium \\ ${ }^{d}$ Department of Material Engineering, KU Leuven, 3001 Leuven, Belgium
}

\begin{abstract}
:
In this paper the sideways self-propulsion behaviour of straight and bent Janus micro-rods is presented. Janus micro-rods are prepared by consecutively sputter-coating Pt and Au on different sides of aligned polystyrene micro-fibers produced via electrospinning. Self-propulsion is induced via the reaction of hydrogen peroxide at the Janus particle interface, and the effect of the particles shape on their self-propulsion trajectories is studied. We show that the self-propulsion trajectories change from straight to circular when the particle shape is changed from a straight to an " $\mathrm{L}$ " shaped rod. In order to understand and quantitatively describe the particle shape effects, we adopted a mathematical model developed by Hagen et al. (2011) to predict their trajectories. We show that the trajectory of irregularly shaped micro-rods depends only on the particle shape. The predicted trajectories for differently shaped particles are in good agreement with the experimental observations.
\end{abstract}




\section{Introduction}

In recent years, understanding the self-propulsion behaviour of biological organisms in order to describe and predict their individual and collective motion in fluids has been gaining importance. The self-propulsion behaviour of such organisms has inspired the creation of artificial selfpropulsion particles that can be controlled and manipulated easily for various applications $[1,2]$. Recently, the self-propulsion of artificial micro- and nanoscale particles has been investigated that convert chemical energy into translational/rotational motion $[2,3]$. In the pioneering work of Whitesides and co-workers [3], they investigated the propulsion of millimetre sized objects (a polydimethylsiloxane (PDMS) plate attached to a platinum $(\mathrm{Pt})$ coated glass plate) by using the catalytic decomposition of hydrogen peroxide on the platinum surface. They observed a propulsion direction away from the $\mathrm{Pt}$ coated side due to the production of oxygen bubbles from the decomposition of hydrogen peroxide on Pt. More recently, self-propulsion of asymmetric nanorods (prepared by the electrodeposition of $\mathrm{Au}$ and Pt inside anodic aluminium oxide) in dilute solutions of hydrogen peroxide was studied by the Mallouk and co-workers [4, 5] where they observed that these nano-rods propelled in the direction of the Pt end of the rod due to a reaction induced electrophoretic mechanism. A schematic diagram of the suggested hydrogen peroxide decomposition reaction on the bimetallic Janus rod is given in Figure 1. This decomposition generates a concentration gradient of oxygen and hydrogen ions across the Pt and Au surface of the particles that is suggested to induce the self-propulsion. However, the exact self-propulsion mechanism of bimetallic coated particle is not yet understood and a number of other possible 
mechanisms such as self-electrophoresis, interfacial tension gradients, bubble recoil, Brownian ratchet mechanisms, and reaction induced charge auto-electrophoresis are discussed [4-9].

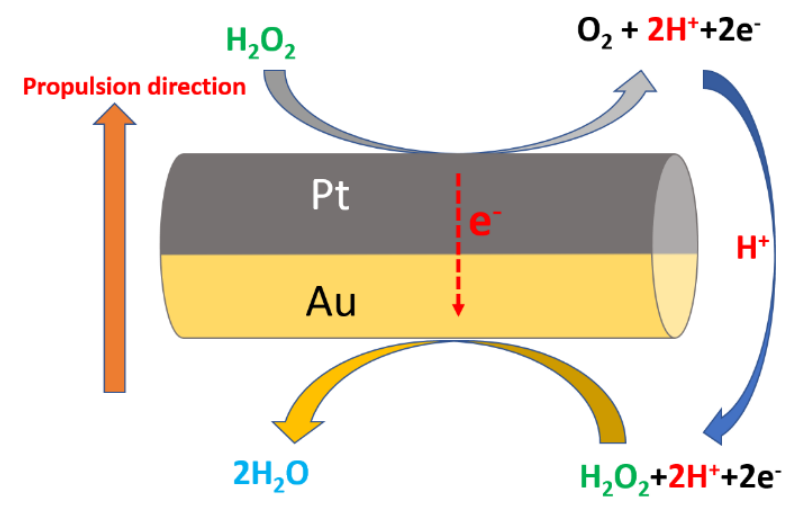

Figure 1. Schematic representation of the hydrogen peroxide decomposition on the $\mathrm{Pt}$ and $\mathrm{Au}$ coated sides of the rod with a reduction of hydrogen peroxide at the Pt and oxidation at the Au side. The propulsion direction is perpendicular to the long-axis of the rod with the Pt side at the front.

Recent developments in the fabrication of micro/nanoscale colloids of complex shapes have expanded the accessible particle shapes beyond the simple rods and have resulted in complex selfpropelled particles such as Janus particles [10-14], stomatocytes [15], multilayer metallic microtubules [16], rollers [17], and active granular particles [3, 18]. Also the driving direction has been altered by modifying the surface pattern [13] or using external fields such as magnetic or light irradiation [19-21]. Recently, Reddy et al. studied the self-propulsion of radially coated bimetallic Janus fibers in hydrogen peroxide solution [13]. In this case the self-propulsion direction is perpendicular to the particle's major axis (as indicated in Figure 1), and different from the case of electrodeposited nanorods [4] where the self-propulsion direction is parallel to the major axis. 
Similarly, the self-propulsion of Janus disks at the interface of hydrogen peroxide solution and air has been studied [14].

The variety of driving mechanisms, ranging from the ones applied in model self-propelled particles to the actual naturally occurring ones in self-propelling organisms, has led to the introduction of many force models in literature to study and describe the single particle [22-25] or the collective motion of particles such as clustering [26, 27], swarming [28-30], and ratchet effects [31, 32]. In general, the relation between the velocity of an anisotropic micro-swimmer particle and the force can be expressed as

$\eta H U=F$

where $\eta$ is the solvent viscosity, $H$ is the grand resistance matrix, $U$ is the generalized velocity (translational and rotational) and $F$ is the generalized force (internal force and torque). This method is widely adopted to describe the motion of microswimmers in 2D and 3D [22, 24, 33, 34]. In general, self-propelling particles are considered as force and torque free because the selfpropulsion force is compensated by the viscous force of the fluid in the absence of any external force and torque. The simultaneous presence of a constant force and torque in the particle frame leads to different trajectories such as circular motion in 2D [22, 34] and helical motion in 3D [24]. Recently, Hagen et al. studied the self-propulsion of anisotropically shaped particles, with and without gravitational effects, and predicted their self-propulsion trajectory by using a slender body approximation model $[23,35,36]$. In particular, they have derived a detailed mathematical model for biaxial diffusiophoretic micro-swimmers and observed a good agreement with their experimental results. They observed that the equation of motion for a self-propulsion particle is the same as for a corresponding passive particle with an effective external force and torque. Furthermore, they applied this model to predict the trajectories of L shaped particle with the self- 
propulsion force acting on only one arm. The applicability of this model is, however, not yet verified for differently shaped particles, especially bent particle with self-propulsion force acting on two arms.

In the present work, we adopt a general mathematical model to predict a micro-swimmer's selfpropulsion trajectory including the effect of anisotropic shapes with a focus on bent rods. These predictions are compared to experimental observations of the side-ways self-propulsion trajectories of straight and bent bi-metallic Janus rods in hydrogen peroxide solutions.

\section{Materials and methods}

\subsection{Fiber production}

Electrospinning was used to produce aligned polystyrene micro-fibers with a narrow distribution of fiber diameters. In this process, a polystyrene solution is ejected through a conducting needle while applying a large DC voltage between the needle and a grounded collector, leading to the formation of a Taylor cone at the end of needle tip. A polymer solution jet ejects out of the Taylor cone, undergoing a further reduction in diameter due to bending instabilities and eventually solidifies into a solid fiber due to fast solvent evaporation. Unidirectional aligned fibers were produced using a rectangular parallel electrode collector as shown in Figure $2[13,37]$. Here, the spinning fiber randomly jumps between the two large collector areas, producing aligned fibers over the gap between the collectors. In the present study, the parallel collector is made of stainless steel with $1 \mathrm{~cm}$ spacing and $4 \mathrm{~cm}$ length. The electrode is placed on the standard circular collector and the remaining area is covered with polytetrafluoroethylene (PTFE) to isolate from the circular collector. Polystyrene (PS, $M_{\mathrm{w}}=280 \mathrm{~kg} / \mathrm{mol}$ ) was dissolved at $30 \mathrm{wt} \%$ in DMF under stirring for

$24 \mathrm{~h}$. Optimized electrospinning experimental conditions are given in Table 1. The alignment of the fibers was shown to be sensitive to the relative humidity and therefore all experiments were 
conducted in a climate controlled electrospinning chamber (EC-CLI, IME Technologies, The Netherlands). Microscopy images of the aligned electro-spun fibers shown in figure 3 were obtained with the Type 1 specifications of Table 1 .

Table 1. Electrospinning experimental conditions and the resulting fiber diameters.

\begin{tabular}{|l|l|l|l|l|l|l|l|}
\hline Type & $\begin{array}{l}\text { Voltage } \\
(\mathrm{kV})\end{array}$ & $\begin{array}{l}\text { Flow } \\
\text { rate } \\
(\mathrm{ml} / \mathrm{min})\end{array}$ & $\begin{array}{l}\text { Needle } \\
\text { inner } \\
\text { diameter } \\
(\mathrm{mm})\end{array}$ & $\begin{array}{l}\text { Temperature } \\
\left({ }^{\circ} \mathrm{C}\right)\end{array}$ & $\begin{array}{l}\text { Relative } \\
\text { humidity }\end{array}$ & $\begin{array}{l}\text { Distance } \\
\text { between the } \\
\text { needle and } \\
\text { collector } \\
(\mathrm{cm})\end{array}$ & $\begin{array}{l}\text { Fiber } \\
\text { diameter } \\
(\mu \mathrm{m})\end{array}$ \\
\hline 1 & 13 & 0.05 & 0.51 & 35 & 23 & 13 & $\sim 6-8$ \\
\hline 2 & 19 & 0.005 & 0.26 & 35 & 26 & 13 & $\sim 2-4$ \\
\hline
\end{tabular}

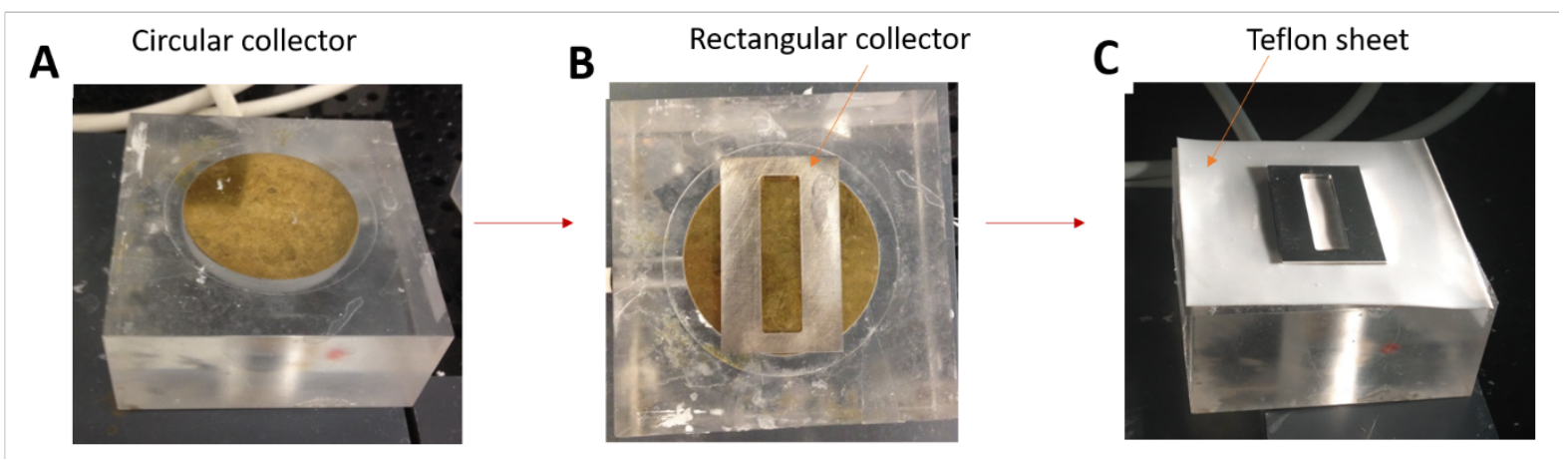

Figure 2. (A) Standard circular collector, (B) Rectangular collector placed on the circular collector for a direct conductive path. (C) the remaining part is covered with the Teflon sheet to isolate the circular collector. The hollow space in the rectangular collector is $4 \mathrm{~cm} \mathrm{x} 0.5 \mathrm{~cm}$. 


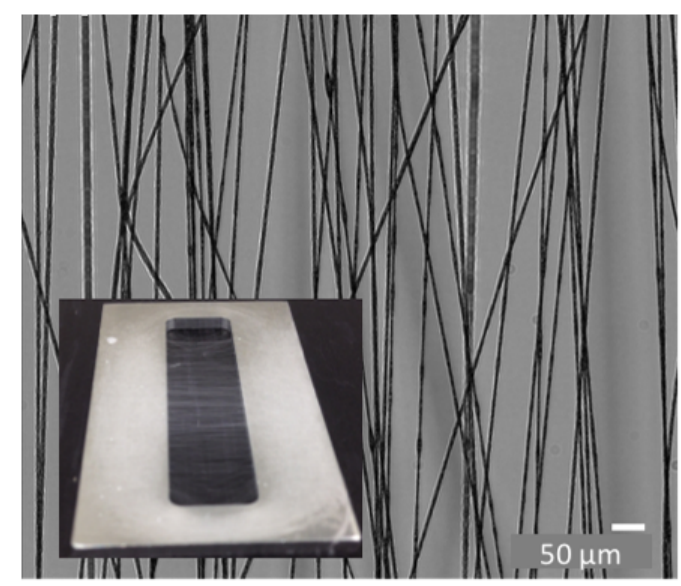

Figure 3. Microscopic image of electrospun, aligned PS fibers with an average diameter of $6 \mu \mathrm{m}$. The inset shows the aligned fibers on the rectangular collectors.

\subsection{Bimetallic coating of aligned fibers and production of micro-rods}

The free standing aligned fibers were coated with $5 \mathrm{~nm}$ thick Pt and Au layers at the top and bottom surface of the fibers respectively (similar to the schematic shown in figure 1) using a sputter coater (Quorum, Q150TS). Due to some overlap (crossing) with other fibers as shown in figure 3, a fraction of the fibers get only partially coated due to shadowing effects. After bi-metallic coating, the fibers were dispersed in water and subsequently sonication for $30 \mathrm{~min}$ to break the fibers. The average length of the fibers depends on the sonication time. A bright field microscopy image of a broken fiber sample is shown in figure 4. In addition to the straight rods, also bent rods are produced in the sonication process due to the creation of defects along the length of the fiber at random points. The obtained particle length and bent shape distribution is given in the supporting information (figure S1 and S2). 


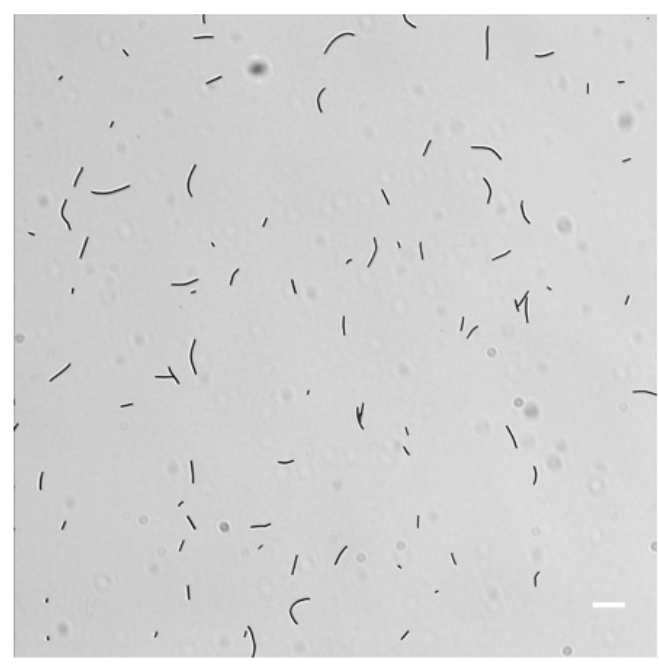

Figure 4. Microscopic image of the broken Janus fibers after sonication, showing short fibers of different shape and size. The scale bar is $50 \mu \mathrm{m}$.

\subsection{Self-propulsion experiments}

Self-propulsion experiments were conducted in 2 and $5 \mathrm{wt} \%$ hydrogen peroxide solutions with two different diameter fibers $(\sim 6 \mu \mathrm{m}$ and $\sim 2 \mu \mathrm{m})$ in a rectangular glass cell $\left(2 \times 2 \times 0.5 \mathrm{~cm}^{3}\right)$. The cell was covered with a cover slip to minimize interface movement. Bright field microscopy (Olympus, BX51WI) at different magnifications (50x, 20x, and 10x) was used to observe the selfpropulsion of particles in $2 \mathrm{D}$ at a rate of $30 \mathrm{fps}$ (focusing only on particles moving close to the bottom of the cell). A particle tracking (MATLAB) algorithm [38] was used to calculate the particle displacement and trajectories. Fibers with non-uniform coating due to the shadow effect either show no self-propulsion or very slow propulsion. For the analysis in this article we have selected only those straight and bent particles that exhibit a consistent, maximum propulsion velocity, assuming those to be fully covered. 


\section{Results}

The self-propulsion behaviour of straight and bent Janus rods was studied at different concentrations of hydrogen peroxide solution. In order to understand the particle shape effects on the self-propulsion trajectory, we will first show the results of the trajectories of straight rods, followed by bent rods with equal arm lengths, and finally bent rods with unequal arm lengths.

\section{Self-propulsion of straight rods}

The self-propulsion of straight rods is set as a benchmark. When these rods are suspended in pure water they show Brownian motion (see movie M1 in the supporting information). However, with the addition of hydrogen peroxide, these rods self-propel, due to the catalytic reaction of $\mathrm{H}_{2} \mathrm{O}_{2}$ on $\mathrm{Pt}$ and $\mathrm{Au}$. The trajectory of a single straight rod in $2 \mathrm{wt} \%$ hydrogen peroxide solution is shown in figure 5 (also see the movie M2 in the supporting information). From the tracking, it is clear that the self-propulsion direction of the straight rod is perpendicular to its long axis. The change in the direction of the straight rod can be attributed, in addition to Brownian motion, to a roughness induced interaction of the particle with the cell bottom. In order to separate the contribution of the Brownian motion from such other factors, we have calculated the mean squared angular displacement (MSAD, see supporting information (figure S4)). From the figure S4, it can be seen that on top of the Brownian motion abrupt and continuous changes in the particle orientation occur that are likely due to random interactions with the cell bottom. 


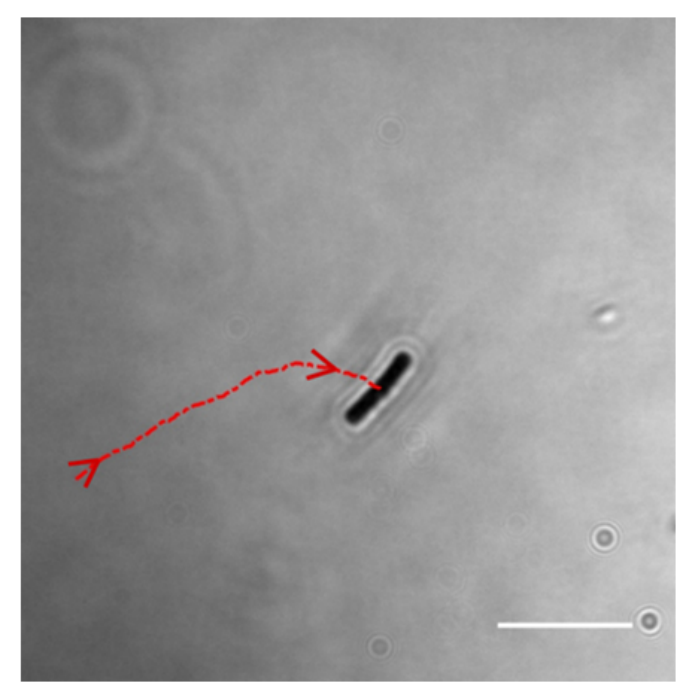

Figure 5. Self-propulsion of a straight rod (length $=13 \mu \mathrm{m}$ and diameter $=2.2 \mu \mathrm{m}$ ) in $2 \mathrm{wt} \%$ hydrogen peroxide solution over a period of $30 \mathrm{~s}$. The particle trajectory (dotted line) shows a straight movement and the propulsion direction is perpendicular to particle major axis. The scale bar is $20 \mu \mathrm{m}$.

In order to show that the translational particle movement in $\mathrm{H}_{2} \mathrm{O}_{2}$ is not governed by the Brownian motion, we calculated the mean squared displacement (MSD) of the straight rods as a function of time. The measured MSD of straight particles of different sizes (diameter and length) is shown in figure 6. The slope of the MSD curves for all observed self-propelling rods is close to 2, which implies non-Brownian motion and superdiffusion $[13,39]$ with an average velocity of $1.6 \mu \mathrm{m} / \mathrm{s}$, in contrast to a purely Brownian motion with a slope equal to 1. 


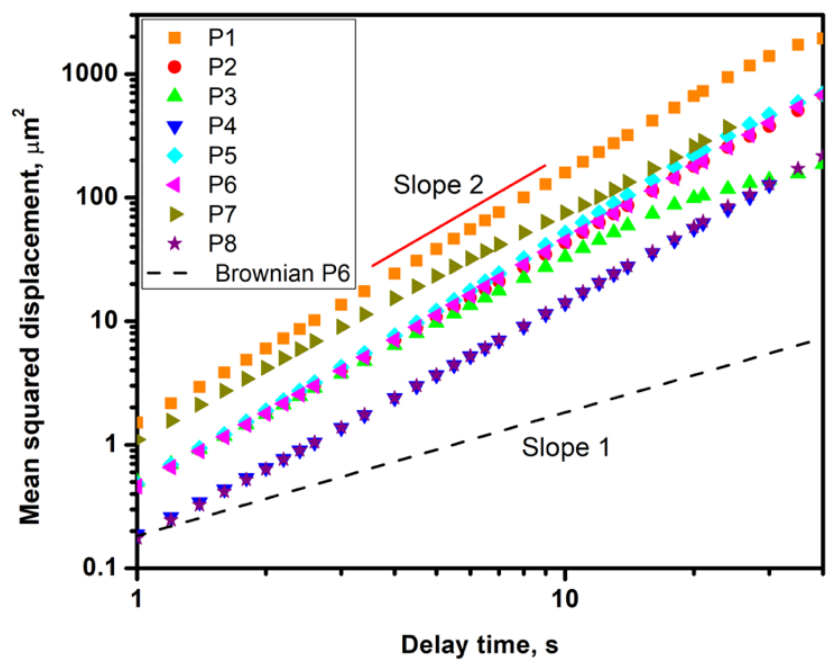

Figure 6. The mean squared displacement (MSD) of different straight rods as a function of time. Filled symbol represent the MSD of self-propelling rods, whereas the dashed line is the calculated MSD of pure Brownian motion for the particle P6. A slope of 2 is obtained for self-propelling rods showing superdiffusion and a slope of 1 for Brownian diffusion.

\subsection{Self-propulsion of bent rods}

The self-propulsion of "L" and "V" shaped particles at different concentrations of hydrogen peroxide is shown in figure 7 and 8 respectively (also see movies M3 and M4 in the supporting information). Different circular and straight trajectories are observed for the "L" and "V" shaped particles, respectively, indicating that the propulsion trajectory depends on the particle shape. To explain the observed particle shape effect on the self-propulsion trajectories, we adopt a mathematical method to predict the trajectory of any irregularly shaped particle. 


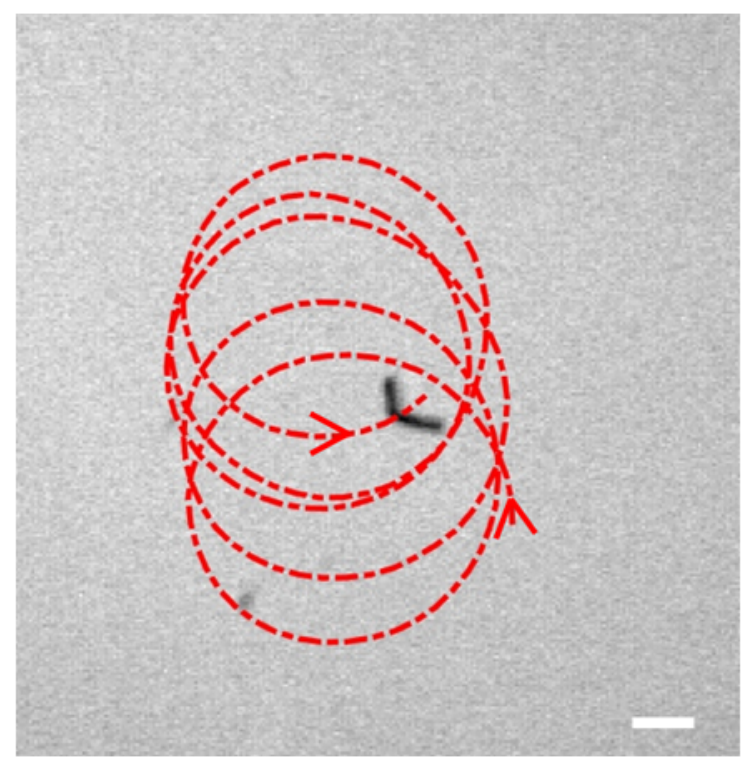

Figure 7. Self-propulsion trajectory (dotted line) of an "L" shaped particle in $5 \mathrm{wt} \%$ hydrogen peroxide solution. The scale bar is $40 \mu \mathrm{m}$.

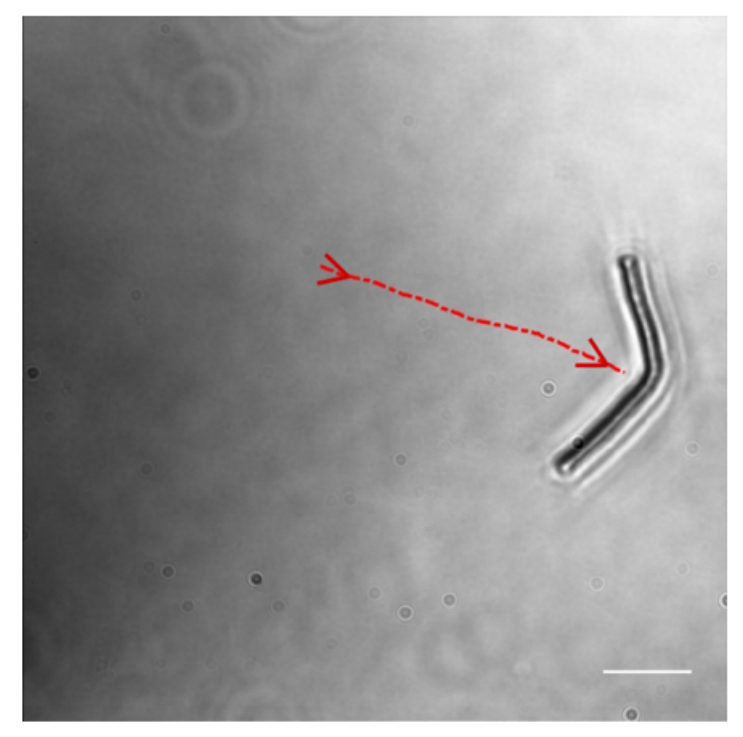

Figure 8. Straight self-propulsion trajectory of a "V" shaped particle in $2 \mathrm{wt} \%$ hydrogen peroxide solution. The scale bar is $20 \mu \mathrm{m}$. 


\section{Modelling and validation}

The self-propulsion of bimetallic coated particles is so far explained by different mechanisms [49]. However, independent of the actual mechanism, the self-propulsion of any microswimmer can be modelled by introducing a non-vanishing slip velocity on the surface of the particle [40, 41]. Recently, Hagen et al. developed a mathematical model to predict the self-propulsion of a simple anisotropic geometry (an 'L-shaped' particle) with and without external forces (gravity) [34-36]. In this model, a leading order slender body approximation (force per unit length) is used to calculate the fluid velocity on the particle surface. Hagen et al. observed that the equation of motion for self-propulsion is the same as for a corresponding passive particle with an effective external force and torque, which can be expressed in $2 \mathrm{D}$ as

$$
\left(\begin{array}{c}
U_{X} \\
U_{Y} \\
\phi
\end{array}\right)=\mathcal{H}^{-1}\left(\begin{array}{c}
F_{X} \\
F_{Y} \\
T
\end{array}\right)
$$

where $U_{X}$ and $U_{Y}$ are the particle velocity in $X$ and $Y$ direction, $\dot{\phi}$ is the particle angular velocity, $\mathcal{H}^{-1}$ is the mobility matrix of the particle, $F_{X}$ and $F_{Y}$ are the self-propulsion forces in the $X$ and $Y$ direction, and $T$ is the torque on the particle acting at the centre of mass. Equation 2 is valid in the Stokes regime for any arbitrary shape of particle.

To use equation 2 , the shape dependent mobility matrix together with the effective torque and force are required to predict the self-propulsion trajectory of any complex particle shape. In order to simplify the problem, the complex particle shape can be described as a combination of spherical beads as shown in figure 9. The corresponding particle mobility matrix can be numerically 
calculated using a beads model $[42,43]$, as analytical equations are either complex or not available for complexly shaped particles. The detailed calculation procedure is explained in Appendix A.
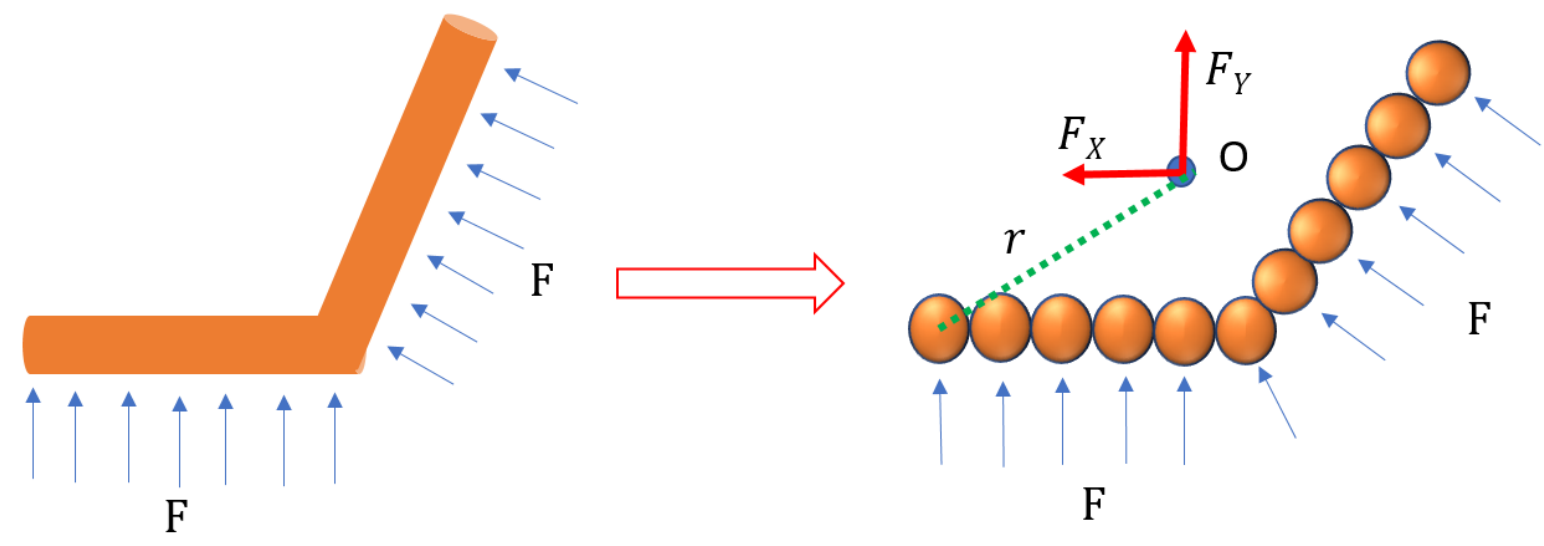

Figure 9. Schematic representation of the bent rod as composed of touching spheres. $F$ is the selfpropulsion force per unit length of the rod. $F_{X}$ and $F_{Y}$ are the net force from all the spheres acting on the rod's centre of mass $(\mathrm{O})$ in the $X$ and $Y$ direction and $r$ is the distance between the individual sphere and the rod's centre of mass.

We can now predicted the particle trajectory and velocity for straight rods by using equation 2 . To calculate the shape dependent mobility matrix, we assumed that there are no wall effects on the particle. The absolute force per unit length of the rod (or per bead in our model) is unknown as this depends on the propulsion mechanism. However, an effective force can be easily determined from the experimentally observed particle velocity. For this we assumed the direction of the selfpropulsion force per unit length to be perpendicular to the long axis of each particle segment (bead) and parallel to the 2D plane in which the particle moves. The total effective force and torque at the particle centre of mass are then obtained by adding the forces for each segment (bead), which means for a straight rod multiplying the particle length with the force per unit length. The self- 
propulsion force is then estimated by using an iterative method, where the particle velocity is calculated using equation 2 with an initial guess of the self-propulsion force, which is then consecutively adjusted until the calculated velocity approaches the experimentally observed average velocity. The obtained effective forces per unit length are shown in figure 10 for different aspect ratios of straight particles. The average of these forces is approximately $0.09 \mathrm{pN}$, which is of similar order of magnitude as other self-propulsion forces reported previously in literature [44]. The self-propulsion force strongly depends on the hydrogen peroxide concentration, particle diameter and length $[9,44,45]$. However, for the rods with bi-metallic coating, particle surface and wall effects also plays an important role that effects self-propulsion force.

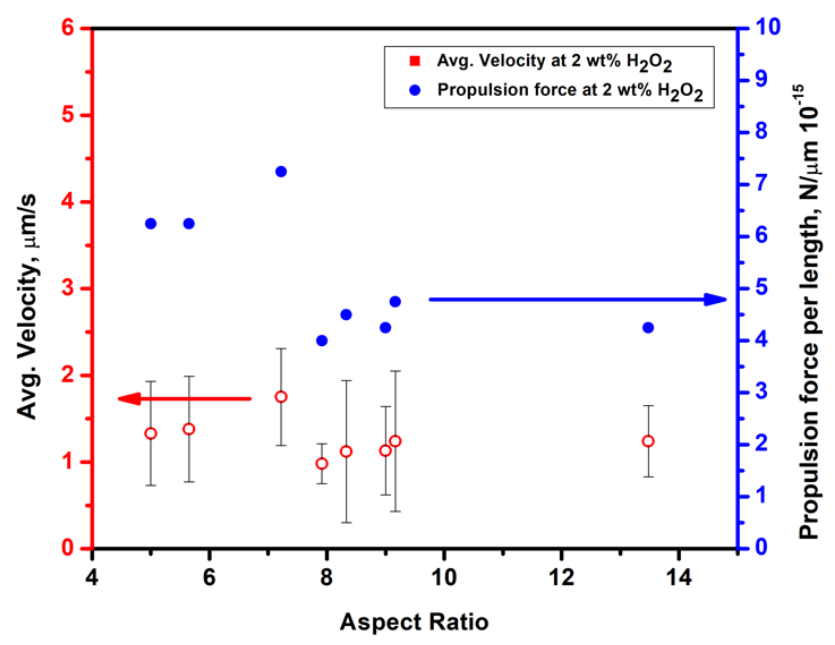

Figure 10. Experimental average velocity and estimated self-propulsion force per unit length for straight rods of different aspect ratios in $2 \mathrm{wt} \%$ hydrogen peroxide solution. The average particle velocity is calculated from the particle mid-point progression over each recorded time interval and the error bars indicate the standard deviation of this averaging. 
Similarly, the trajectories of bent particles are calculated following the same procedure as for the straight rods. The experimental and model trajectories for "L" and "V" shaped particle are shown in figure 11 and 12 respectively. The estimated self-propulsion force per unit length of the "L" shaped particle in $5 \mathrm{wt} \%$ hydrogen peroxide solution is $1.05 * 10^{-13} \mathrm{~N} / \mu \mathrm{m}$, with an estimated trajectory diameter of $186 \mu \mathrm{m}$, which is in good agreement with the experimental observation of $177 \mu \mathrm{m}$. The estimated force per unit length for the "V" shaped particle in $2 \mathrm{wt} \%$ hydrogen peroxide solution is $6.5 * 10^{-15} \mathrm{~N} / \mu \mathrm{m}$, which is of the same order of magnitude as for straight rods at the same hydrogen peroxide concentration.

In order to verify the proposed method, we compare experimental results of self-propulsion trajectories for a number of different, slightly bent rods with the model predictions, using the respective average force per unit length obtained above. The predication are in good agreement with the experimental observations and the results are shown in the supporting information (S6 and S7). The self-propulsion trajectory diameter $R$ can be calculated from $R=U / \dot{\phi}$

where $U$ is the predicted translational velocity and $\dot{\phi}$ is the angular velocity. The thus determined trajectory diameter of the different bent particle is given in Table 2. 
Table 2: Model predicted trajectory diameter for bent rods with different bending angles.

\begin{tabular}{|l|l|l|l|l|l|l|l|l|}
\hline & $\begin{array}{l}\text { Arm } \\
(\mathrm{L} 1) \\
(\mu \mathrm{m})\end{array}$ & $\begin{array}{l}\text { Arm } \\
(\mu \mathrm{L}) \\
(\mu \mathrm{m})\end{array}$ & $\begin{array}{l}\text { Particle } \\
\text { diameter }\end{array}$ & $\begin{array}{l}\text { Angle } \\
\text { between }\end{array}$ & $\begin{array}{l}\mathrm{H}_{2} \mathrm{O}_{2} \\
(\mathrm{wt} \%)\end{array}$ & $\begin{array}{l}\text { Translation } \\
\text { Velocity }\end{array}$ & $\begin{array}{l}\text { Angular } \\
\text { Velocity } \\
(\mathrm{rad} / \mathrm{s})\end{array}$ & $\begin{array}{l}\text { Predicted } \\
\text { trajectory } \\
\text { diameter } \\
(\mu \mathrm{m})\end{array}$ \\
\hline Fig 11 & 20 & 30 & 4.75 & 105 & 5 & 27.58 & 0.29 & 186 \\
\hline Fig 12 & 26 & 29 & 2.00 & 120 & 2 & 1.95 & $3.60 \times 10^{-3}$ & $1.07 \times 10^{3}$ \\
\hline Fig S6 & 14 & 25 & 2.00 & 160 & 2 & 1.50 & $0.35 \times 10^{-3}$ & $8.42 \times 10^{3}$ \\
\hline Fig S7 & 56 & 112 & 5.00 & 162 & 4 & 13.69 & $2.10 \times 10^{-3}$ & $13.3 \times 10^{3}$ \\
\hline
\end{tabular}

From the modelling and experiments, it is evident that the particle velocity depends on the selfpropulsion force (hydrogen peroxide concentration, catalyst and particle size) and the drag (particle size and speed, viscosity and wall effects), whereas the particle trajectory depends only on the particle shape. In case of two arm particles, the difference in the length of the arms determines the trajectory type (straight or circular) and the trajectory diameter depends on the angle between the arms and the length of the arms. For the case of the L shaped particles studied in this work, the unequal length of the particle arms generates a net torque on the particle's centre of mass, which rotates the particle. In case of a $\mathrm{V}$ shaped particle, the equal length of the two arms creates a zero net torque, so that the particle moves in one direction without rotation. In spite of the many assumptions, the proposed model is capable of predicting the self-propulsion parameters such as the self-propulsion force and trajectory for any arbitrarily shaped particles and is qualitatively and quantitatively in good agreement with the experimental observations. Future 
work will include wall effects in the mobility matrix to further improve the model predictions to the experimental finding.
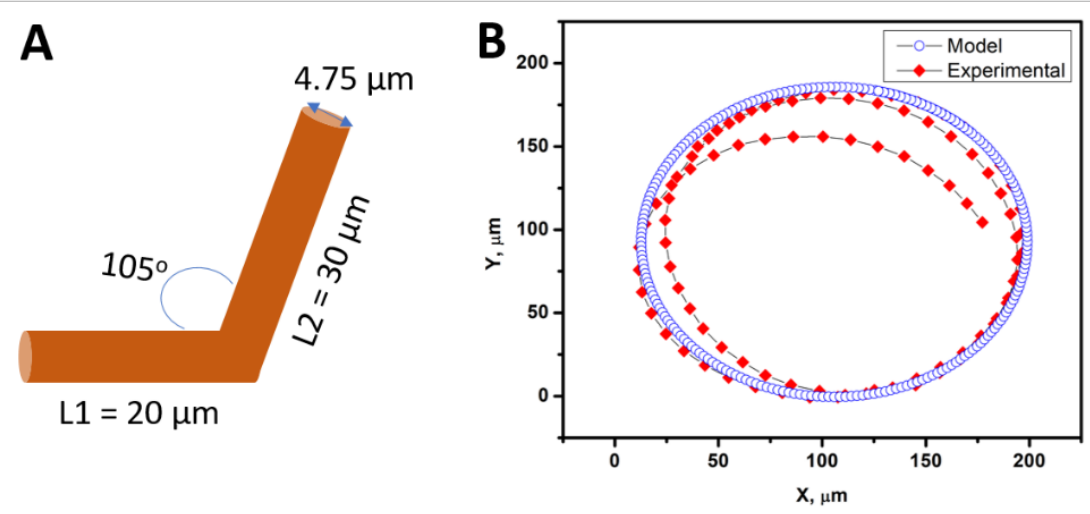

Figure 11. A) Physical dimensions of the "L" shaped particle. B) Predicted and experimental selfpropulsion trajectories of the " $\mathrm{L}$ " shaped particle in $5 \mathrm{wt} \% \mathrm{H}_{2} \mathrm{O}_{2}$ solution. The estimated force per unit length is $1.05 * 10^{-13} \mathrm{~N} / \mu \mathrm{m}$.
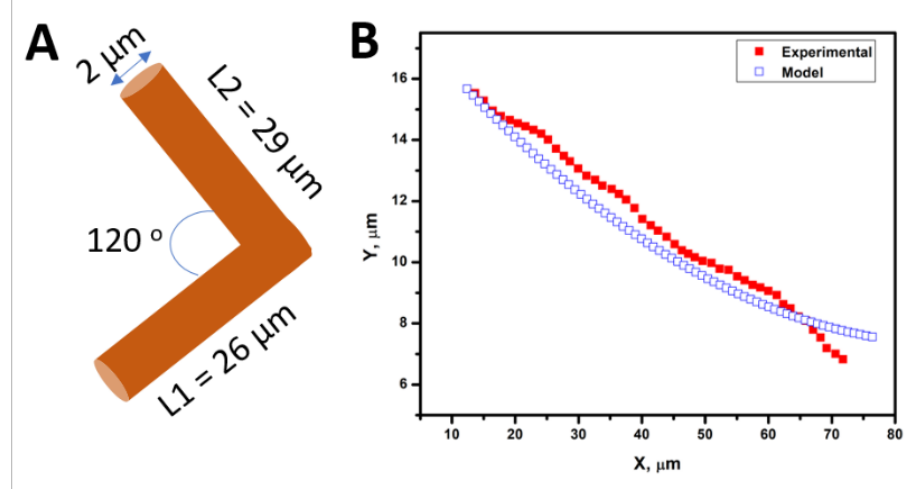

Figure 12. A) Physical dimensions of the "V" shaped particle. B) Self-propulsion trajectory predicted from the model and from experiments at $2 \mathrm{wt} \% \mathrm{H}_{2} \mathrm{O}_{2}$ solution. The estimated force per unit length is $6.5 * 10^{-15} \mathrm{~N} / \mu \mathrm{m}$. 


\section{Conclusion}

In this work, we studied the side-ways self-propulsion behaviour of bimetallic Janus rods with irregular shapes. Straight trajectories are observed for straight rods and 'V' shaped rods, whereas circular trajectories are observed for "L" shaped rods. We have adopted a mathematical model based on Hagen et al. (2011) to predict the self-propulsion trajectories of straight and bent Janus rods. The model predictions are in good agreement with the experimental observation and allow to extract the average force per unit length acting on the Janus rods. From the model and experiments it is clear that the particle trajectory only depends on particle shape. The main advantage of this model is that it can predict both qualitatively and quantitatively the selfpropulsion parameters for any complexly shaped particles in $2 \mathrm{D}$ and $3 \mathrm{D}$ if the particles mobility matrix is known. Future research will focus on the controlled bending of bimetallic particles using techniques such as laser etching to study the collective behaviour of self-propelled bent rods.

\section{Appendix A:}

\section{Calculation of the diffusion matrix of irregularly shaped particle:}

In this section we explain the calculation procedure for the diffusion matrix of an irregularly shaped particle. In the Stokes's regime, if the particle moves with a translational velocity $u_{O}$ and angular velocity $\omega$, the friction force and torque experienced by the particle are

$$
\begin{aligned}
& F=\Xi^{t t} \cdot u_{O}+\Xi_{O}^{t r} \cdot \omega \\
& T_{O}=\Xi_{O}^{r t} \cdot u_{O}+\Xi_{O}^{r r} \cdot \omega
\end{aligned}
$$

Here, $\Xi$ 's are the $3 \times 3$ friction matrices for translation $(t t)$, rotation $(r r)$ and translation-rotation coupling $(t r, r t)$. The analytical expressions of the friction matrix for simple shapes such as for spheres and rods are available in literature. However, an analytical expression for the friction 
matrix of an irregular shape is much more complicated to obtain. In order to calculate a friction matrix tensor of an irregular particle, it is often approximated by a beads model [42, 43]. In a beads model, the irregularly shaped particle can be represented as a rigid assembly of touching beads, as shown in figure 9. If the rigid assembly of spheres moves in a fluid, all rigid beads move with an overall translational velocity $u_{O}$ with respect to an origin $\mathrm{O}$ (in the current case the particle's centre of mass), and a rotational velocity $\omega$. The angular velocity of all the beads is $\omega_{i}=\omega$, and the linear velocity of their centre points is $u_{i}=u_{O}+\omega \times r_{i}$, where $r_{i}$ is the bead's position vector from the origin $\mathrm{O}$. The friction force and torque experienced by the individual bead depend on the force exerted by the neighbouring beads. The friction force and torque of an individual bead is then

$$
\begin{aligned}
& F_{i}=\sum_{j=1}^{N} \zeta_{i j}^{t t} \cdot\left(u_{O}+\omega \times r_{j}\right)+\left(\sum_{j=1}^{N} \zeta_{i j}^{t r}\right) \cdot \omega \\
& T_{P i}=\sum_{j=1}^{N} \zeta_{i j}^{r t} \cdot\left(u_{O}+\omega \times r_{j}\right)+\left(\sum_{j=1}^{N} \zeta_{i j}^{r r}\right) \cdot \omega
\end{aligned}
$$

where $\zeta_{i j}$ 's are the $3 \times 3$ friction matrices between two beads. As for the Stokes's linearity, the total force and torque on the rigid body is the sum of the individual bead's forces and torques. With this the total force and torque of the rigid body is

$$
\begin{aligned}
& F=\sum_{i=1}^{N} F_{i}=\Xi^{t t} \cdot u_{O}+\Xi_{O}^{t r} \cdot \omega \\
& T_{O}=\sum_{i=1}^{N} T_{i}=\Xi_{O}^{r t} \cdot u_{O}+\Xi_{O}^{r r} \cdot \omega
\end{aligned}
$$

where

$$
\begin{aligned}
& \Xi^{t t}=\sum_{i=1}^{N} \sum_{j=1}^{N} \zeta_{i j}^{t t} \\
& \Xi_{O}^{t r}=\sum_{i=1}^{N} \sum_{j=1}^{N}\left(-\zeta_{i j}^{t t} \cdot A_{j}+\zeta_{i j}^{t r}\right) \\
& \Xi_{O}^{r t}=\sum_{i=1}^{N} \sum_{j=1}^{N}\left(\zeta_{i j}^{r t}+A_{i} \cdot \zeta_{i j}^{t t}\right) \\
& \Xi_{O}^{r r}=\sum_{i=1}^{N} \sum_{j=1}^{N}\left(\zeta_{i j}^{r r}-\zeta_{i j}^{r t} \cdot A_{j}+A_{i} \cdot \zeta_{i j}^{t r}-A_{i} \cdot \zeta_{i j}^{t t} \cdot A_{j}\right)
\end{aligned}
$$


and where the $\Xi$ s are translation, rotation and coupling friction matrices of a rigid body and $A$ is the Levi-Civita tensor. The detailed derivation of the friction tensor for a rigid body using this beads model is given by Carrasco et al [43]. In order to calculate the friction matrix of an irregular particle via the beads model, first we need to calculate the grand mobility matrix between the beads. There are different analytical expressions available in literature to calculate such a mobility matrix [43]. In the present work, we used the Reuland-Felderhoh-Jones expression, which includes multi-body interactions to reduce the error [43]. Following this, the grand mobility matrix of a rigid bead is

$$
\left(\begin{array}{ll}
\mu^{t t} & \mu^{t r} \\
\mu^{r t} & \mu^{r r}
\end{array}\right)=\left(\begin{array}{cccccc}
\mu_{11}^{t t} & \cdots & \mu_{1 N}^{t t} & \mu_{11}^{t r} & \cdots & \mu_{1 N}^{t r} \\
\vdots & \ddots & \vdots & \vdots & \ddots & \vdots \\
\mu_{N 1}^{t t} & \cdots & \mu_{N N}^{t t} & \mu_{N 1}^{t r} & \cdots & \mu_{N N}^{t r} \\
\mu_{11}^{r t} & \cdots & \mu_{1 N}^{r t} & \mu_{11}^{r r} & \cdots & \mu_{1 N}^{r r} \\
\vdots & \ddots & \vdots & \vdots & \ddots & \vdots \\
\mu_{N 1}^{r t} & \cdots & \mu_{N N}^{r t} & \mu_{N 1}^{r r} & \cdots & \mu_{N N}^{r r}
\end{array}\right)
$$

where $N$ is the number of touching beads. From the grand mobility matrix, we calculated the grand friction matrix by inverting the mobility matrix. With the grand friction matrix the friction tensor of the rigid particle is calculated using equations (A7) - (A10). From the friction tensor, the diffusion matrix of a rigid body is calculated using the Stokes-Einstein equation

$$
D=\frac{k_{B} T}{\eta}\left(\begin{array}{ll}
\Xi^{t t} & \Xi_{O}^{t r} \\
\Xi_{O}^{r t} & \Xi_{O}^{r r}
\end{array}\right)^{-1}
$$

where $D$ is the diffusion matrix, $k_{\mathrm{B}}$ is the Boltzmann constant, $T$ is the solvent temperature and $\eta$ is the solvent viscosity. The diffusion matrix of a rigid particle in $2 \mathrm{D}$ is

$$
D=\left(\begin{array}{lll}
D_{X X} & D_{X Y} & D_{X R} \\
D_{Y X} & D_{Y Y} & D_{Y R} \\
D_{R X} & D_{R Y} & D_{R R}
\end{array}\right)
$$


Here, the subscripts $\mathrm{X}$ and $\mathrm{Y}$ indicate the diffusivity of particle in $\mathrm{X}$ and $\mathrm{Y}$ direction, respectively, and the subscript $\mathrm{R}$ indicates the rotational diffusivity. The calculated diffusion values of straight and bent rods by using the beads model are given in Table (A1).

Table A1. Diffusion values of straight and bent rods.

\begin{tabular}{|c|c|c|c|}
\hline Diffusion values & $\begin{array}{l}\text { Straight rod } \\
\mathrm{L}=13 \mu \mathrm{m}, \mathrm{D}=2.2 \mu \mathrm{m}^{2}\end{array}$ & $\begin{array}{l}\text { L shape } \\
\mathrm{L} 1=20 \mu \mathrm{m} \mathrm{L} 2=30 \mu \mathrm{m} \\
\mathrm{D}=4.75 \mu \mathrm{m}, \theta=105^{\circ}\end{array}$ & $\begin{array}{l}\text { V shape } \\
\mathrm{L} 1=26 \mu \mathrm{m} \mathrm{L} 2=29 \mu \mathrm{m} \\
\mathrm{D}=2 \mu \mathrm{m}, \theta=120^{\circ}\end{array}$ \\
\hline$D_{X X}, \mu \mathrm{m}^{2} / \mathrm{s}$ & 0.0943 & 0.0283 & 0.0313 \\
\hline$D_{Y Y}, \mu \mathrm{m}^{2} / \mathrm{s}$ & 0.0705 & 0.0305 & 0.0344 \\
\hline$D_{X Y}, \mu \mathrm{m}^{2} / \mathrm{s}$ & 0 & 0.0024 & $3.4504 * 10^{-4}$ \\
\hline$D_{R R}, 1 / \mathrm{s}$ & 0.0079 & $2.2504 * 10^{-4}$ & $1.3670 * 10^{-4}$ \\
\hline$D_{R X}, \mu \mathrm{m} / \mathrm{s}$ & 0 & $1.5060 * 10^{-4}$ & $4.2426 * 10^{-5}$ \\
\hline$D_{R Y}, \mu \mathrm{m} / \mathrm{s}$ & 0 & $9.8551 * 10^{-4}$ & $1.3518 * 10^{-4}$ \\
\hline
\end{tabular}

\section{Acknowledgments}

This work was supported by the Research Foundation - Flanders (FWO) grant no. G077916N, and DVR acknowledges support from KU Leuven Internal Funds (PDM/16/123) 


\section{Reference:}

[1] Wang J and Gao W 2012 Nano/microscale motors: biomedical opportunities and challenges ACS nano 6 5745-51

[2] Ebbens S J and Howse J R 2010 In pursuit of propulsion at the nanoscale Soft Matter 6 726-38

[3] Ismagilov R F, Schwartz A, Bowden N and Whitesides G M 2002 Autonomous Movement and Self-Assembly Angew. Chem. 114 674-6

[4] Paxton W F, Kistler K C, Olmeda C C, Sen A, St. Angelo S K, Cao Y, Mallouk T E, Lammert P E and Crespi V H 2004 Catalytic nanomotors: autonomous movement of striped nanorods J. Am. Chem. Soc. 126 13424-31

[5] Paxton W F, Sen A and Mallouk T E 2005 Motility of Catalytic Nanoparticles through Self-Generated Forces Chemistry-A European Journal $116462-70$

[6] Fournier-Bidoz S, Arsenault A C, Manners I and Ozin G A 2005 Synthetic self-propelled nanorotors Chem. Commun. 441-3

[7] Dhar P, Fischer T M, Wang Y, Mallouk T, Paxton W and Sen A 2006 Autonomously moving nanorods at a viscous interface Nano Lett. 6 66-72

[8] Wang Y, Hernandez R M, Bartlett D J, Bingham J M, Kline T R, Sen A and Mallouk T E 2006 Bipolar electrochemical mechanism for the propulsion of catalytic nanomotors in hydrogen peroxide solutions Langmuir 22 10451-6

[9] Moran J L and Posner J D 2011 Electrokinetic locomotion due to reaction-induced charge auto-electrophoresis J. Fluid Mech. 680 31-66

[10] Theurkauff I, Cottin-Bizonne C, Palacci J, Ybert C and Bocquet L 2012 Dynamic clustering in active colloidal suspensions with chemical signaling Phys. Rev. Lett. 108 268303

[11] Buttinoni I, Bialké J, Kümmel F, Löwen H, Bechinger C and Speck T 2013 Dynamical clustering and phase separation in suspensions of self-propelled colloidal particles Phys. Rev. Lett. 110238301

[12] Wang W, Duan W, Sen A and Mallouk T E 2013 Catalytically powered dynamic assembly of rod-shaped nanomotors and passive tracer particles Proc. Natt. Acad. Sci. 110 17744-9

[13] Reddy N K, Palangetic L, Stappers L, Buitenhuis J, Fransaer J and Clasen C 2013 Metallic and bi-metallic Janus nanofibers: electrical and self-propulsion properties J. Mater. Chem. C $13646-50$

[14] Reddy N K and Clasen C 2014 Self-propelling micro-disks Korea-Aust. Rheol. J. 26 73-9

[15] Wilson D A, Nolte R J and Van Hest J C 2012 Autonomous movement of platinum-loaded stomatocytes Nat. Chem. 4 268-74

[16] Sanchez S, Solovev A A, Harazim S M and Schmidt O G 2010 Microbots swimming in the flowing streams of microfluidic channels J. Am. Chem. Soc. 133 701-3

[17] Bricard A, Caussin J-B, Desreumaux N, Dauchot O and Bartolo D 2013 Emergence of macroscopic directed motion in populations of motile colloids Nature 503 95-8

[18] Narayan V, Ramaswamy S and Menon N 2007 Long-lived giant number fluctuations in a swarming granular nematic Science 317 105-8

[19] Mou F, Pan D, Chen C, Gao Y, Xu L and Guan J 2015 Magnetically Modulated Pot-Like MnFe2O4 Micromotors: Nanoparticle Assembly Fabrication and their Capability for Direct Oil Removal Adv. Funct. Mater. 25 6173-81 
[20] Chen C, Mou F, Xu L, Wang S, Guan J, Feng Z, Wang Q, Kong L, Li W and Wang J 2017 Light-Steered Isotropic Semiconductor Micromotors Adv. Mater. 291603374

[21] Mou F, Li Y, Chen C, Li W, Yin Y, Ma H and Guan J 2015 Single-Component TiO2 Tubular Microengines with Motion Controlled by Light-Induced Bubbles Small 11 256470

[22] van Teeffelen S and Löwen H 2008 Dynamics of a Brownian circle swimmer Phys. Rev. E 78020101

[23] ten Hagen B, van Teeffelen S and Löwen H 2011 Brownian motion of a self-propelled particle J. Phys.: Condens. Matter 23194119

[24] Wittkowski R and Löwen H 2012 Self-propelled Brownian spinning top: dynamics of a biaxial swimmer at low Reynolds numbers Phys. Rev. E 85021406

[25] Elgeti J and Gompper G 2013 Wall accumulation of self-propelled spheres EPL (Europhysics Letters) 10148003

[26] Peruani F, Deutsch A and Bär M 2006 Nonequilibrium clustering of self-propelled rods Phys. Rev. E 74030904

[27] Wensink H and Löwen H 2008 Aggregation of self-propelled colloidal rods near confining walls Phys. Rev. E 78031409

[28] Chen H-Y and Leung K-t 2006 Rotating states of self-propelling particles in two dimensions Phys. Rev. E 73056107

[29] Li Y-X, Lukeman R and Edelstein-Keshet L 2008 Minimal mechanisms for school formation in self-propelled particles Physica D: Nonlinear Phenomena 237 699-720

[30] Wensink H and Löwen H 2012 Emergent states in dense systems of active rods: from swarming to turbulence J. Phys.: Condens. Matter 24464130

[31] Angelani L, Costanzo A and Di Leonardo R 2011 Active ratchets EPL (Europhysics Letters) 9668002

[32] Reichhardt C and Reichhardt C O 2013 Active matter ratchets with an external drift Phys. Rev. E 88062310

[33] Radtke P K and Schimansky-Geier L 2012 Directed transport of confined Brownian particles with torque Phys. Rev. E 85051110

[34] Kümmel F, ten Hagen B, Wittkowski R, Buttinoni I, Eichhorn R, Volpe G, Löwen H and Bechinger C 2013 Circular motion of asymmetric self-propelling particles Phys. Rev. Lett. 110198302

[35] Ten Hagen B, Kümmel F, Wittkowski R, Takagi D, Löwen H and Bechinger C 2014 Gravitaxis of asymmetric self-propelled colloidal particles Nature communications $\mathbf{5} 4829$

[36] Ten Hagen B, Wittkowski R, Takagi D, Kümmel F, Bechinger C and Löwen H 2015 Can the self-propulsion of anisotropic microswimmers be described by using forces and torques? J. Phys.: Condens. Matter 27194110

[37] Li D, Wang Y and Xia Y 2004 Electrospinning nanofibers as uniaxially aligned arrays and layer-by-layer stacked films Adv. Mater. 16 361-6

[38] Kelley D H and Ouellette N T 2011 Using particle tracking to measure flow instabilities in an undergraduate laboratory experiment Am. J. Phys. 79 267-73

[39] Vutukuri H R, Preisler Z, Besseling T H, Van Blaaderen A, Dijkstra M and Huck W T 2016 Dynamic self-organization of side-propelling colloidal rods: experiments and simulations Soft matter 12 9657-65

[40] Anderson J L 1989 Colloid transport by interfacial forces Annu. Rev. fluid mechanics 21 61-99 
[41] Ajdari A and Bocquet L 2006 Giant amplification of interfacially driven transport by hydrodynamic slip: Diffusio-osmosis and beyond Phys. Rev. Lett. 96186102

[42] Riseman J and Kirkwood J G 1950 The Intrinsic Viscosity, Translational and Rotatory Diffusion Constants of Rod-Like Macromolecules in Solution J. Chem. Phys. 18 512-6

[43] Carrasco B and Garcia de la Torre J 1999 Improved hydrodynamic interaction in macromolecular bead models $J$. Chem. Phys. 111 4817-26

[44] Burdick J, Laocharoensuk R, Wheat P M, Posner J D and Wang J 2008 Synthetic nanomotors in microchannel networks: Directional microchip motion and controlled manipulation of cargo J. Am. Chem. Soc. $1308164-5$

[45] Moran J, Wheat P and Posner J 2010 Locomotion of electrocatalytic nanomotors due to reaction induced charge autoelectrophoresis Phys. Rev. E 81065302 


\title{
Supporting Information
}

\section{Self-propulsion of bent bimetallic Janus rods}

\author{
Dugyala Venkateshwar Rao ${ }^{\mathrm{a}}$, Naveen Reddy,c, Jan Fransaer ${ }^{\mathrm{d}}$ and Christian Clasen ${ }^{\mathrm{a} *}$ \\ a Department of Chemical Engineering, KU Leuven, 3001 Leuven, Belgium \\ ${ }^{b}$ Hasselt University, Martelarenlaan 42, 3500 Hasselt, Belgium \\ c IMO-IMOMEC, Wetenschapspark 1, 3590 Diepenbeek, Belgium \\ ${ }^{d}$ Department of Material Engineering, KU Leuven, 3001 Leuven, Belgium
}

\section{Particle size distribution:}

Sonication is used to break the bimetallic coated aligned fibers. The distribution of rod lengths and bending angles between two arm of the bent particles are shown in figures S1 and S2.

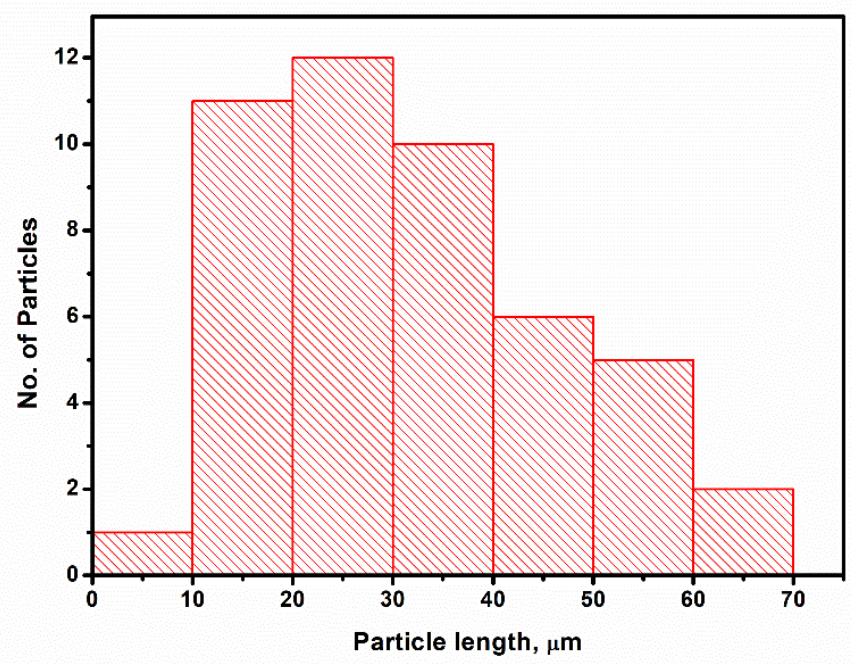

Figure S1: Particle length distribution after sonication. 


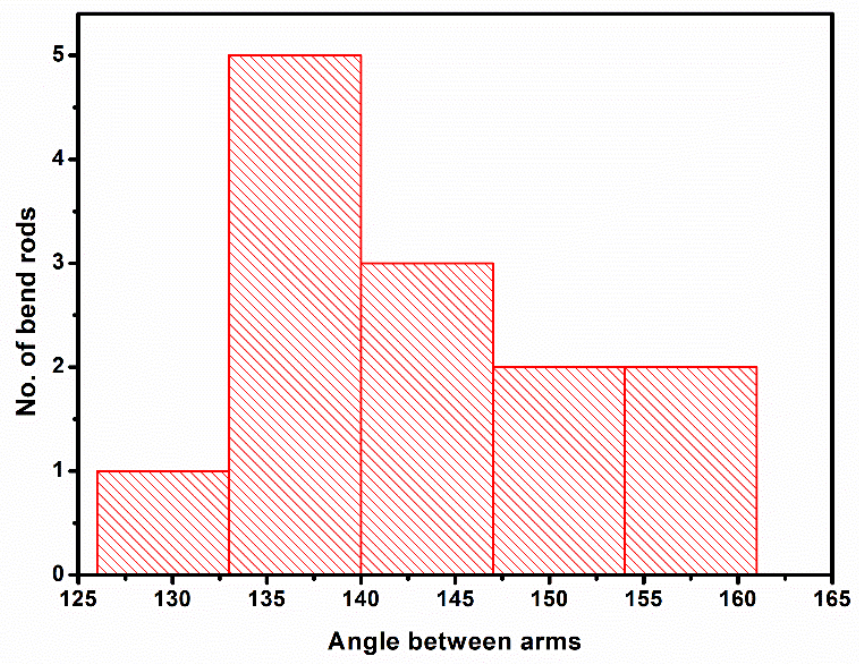

Figure S2: Angle distribution of angles between the two arms of bent rods after the sonication process.

\section{Mean squared angular displacement of straight rod}

The rotational angle of the particle's major axis (figure 5) with respect to the $\mathrm{x}$-axis is shown in Figure S3. From that, the mean squared angular displacement (MSAD) of the particle is calculated, as well as theoretically the MSAD for a purely Brownian motion of the same particle, and both are shown in figure S4. Initially the MSAD of the self-propelling particle exhibits a straight line as the Brownian particle, but then suddenly deviates at the later times, which is due to a roughness induced interaction of the particle with the cell bottom. In order to quantify the difference between the rotational diffusion of self-propulsion particle and pure Brownian particle, the rotational diffusion of self-propulsion particle is calculated by fitting the initial MSAD data (figure S5). The rotational diffusion of the self-propulsion particle is $0.3256 \mathrm{rad}^{2} / \mathrm{s}$ whereas that of the pure Brownian particle is $0.0022 \mathrm{rad}^{2} / \mathrm{s}$. 


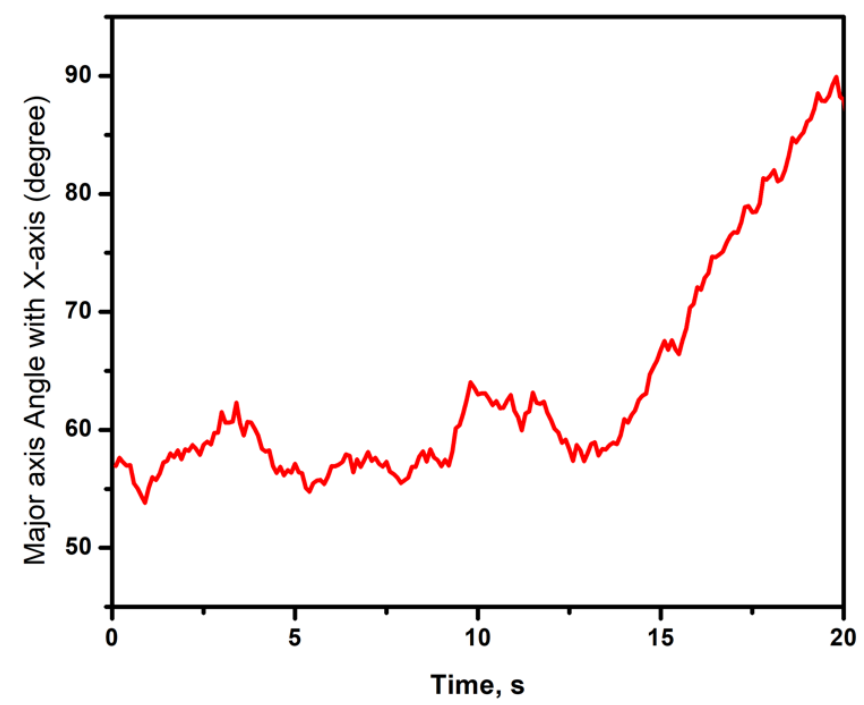

Figure S3: Rotation angle of particle's major axis with respect to the $\mathrm{X}$-axis.

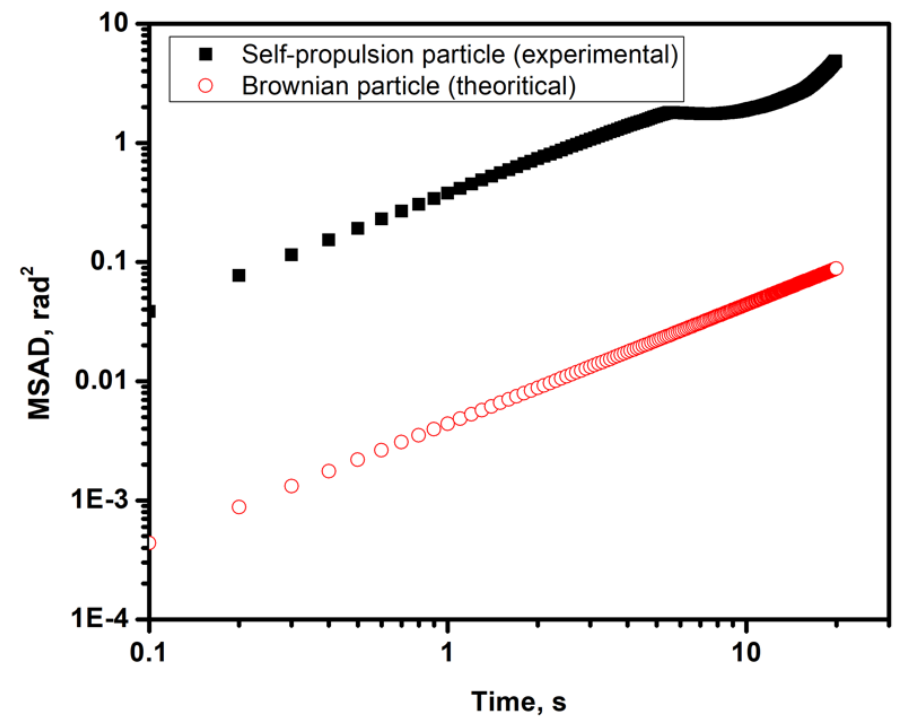

Figure S4: Mean squared angular displacement (MSAD) of self-propelling particle (filled square) and pure Brownian particle (open circle) as a function of time. The mean squared angular displacement (MSAD) of the pure Brownian particle is calculated theoretically. Self-propulsion particle is showing a non-Brownian motion at later stages. 


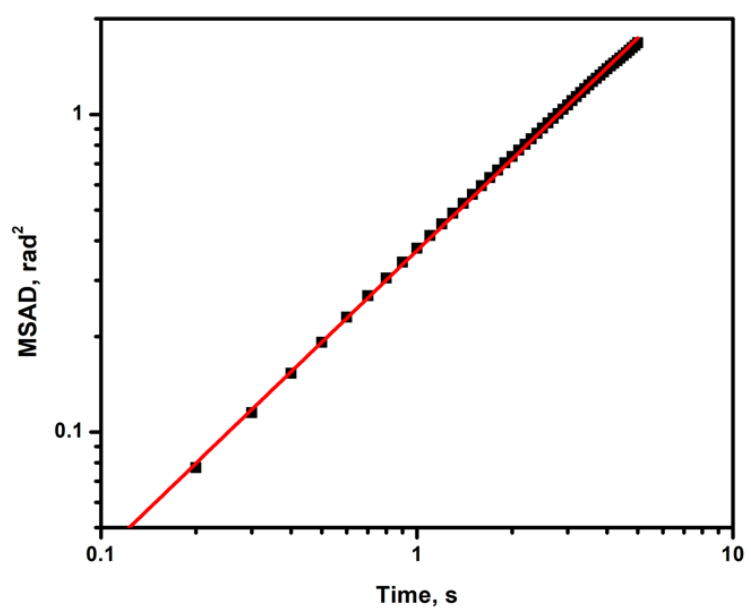

Figure S5: The rotational diffusion of the self-propulsion particle is calculated by fitting the initial MSAD data. The fit is shown as a solid line. The obtained rotational diffusion of the selfpropelling particle is $0.3256 \mathrm{rad}^{2} / \mathrm{s}$.

\section{Self-propulsion of bent rods:}

Additional experimental self-propulsion trajectories of different slightly bent rods are compared to the theoretically predicted ones by the proposed method are shown in figures S6 and S7.
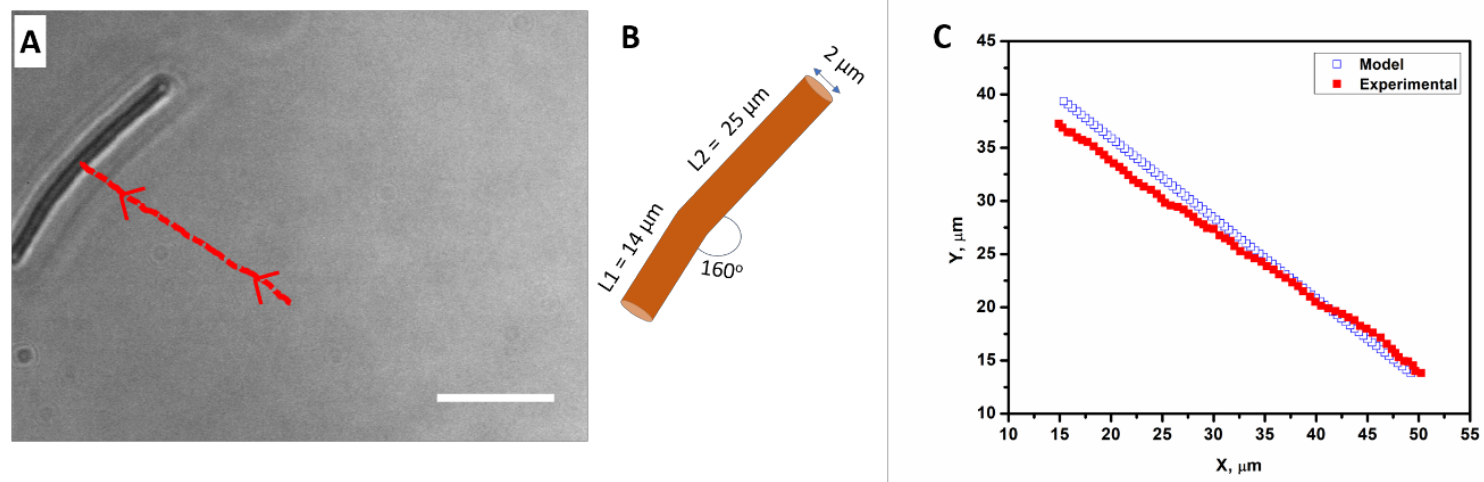

Figure S5. (A) Self-propulsion trajectory of a bent rod in $2 \mathrm{wt} \%$ hydrogen peroxide solution with an average velocity of $1.50 \pm 0.44 \mu \mathrm{m} / \mathrm{s}$. The scale bar is $20 \mu \mathrm{m}$. B) Physical dimensions of the 
bent rod. C) Predicted and experimental self-propulsion trajectories of the bent rod. The estimated force per unit length is $4.5 * 10^{-15} \mathrm{~N} / \mu \mathrm{m}$.
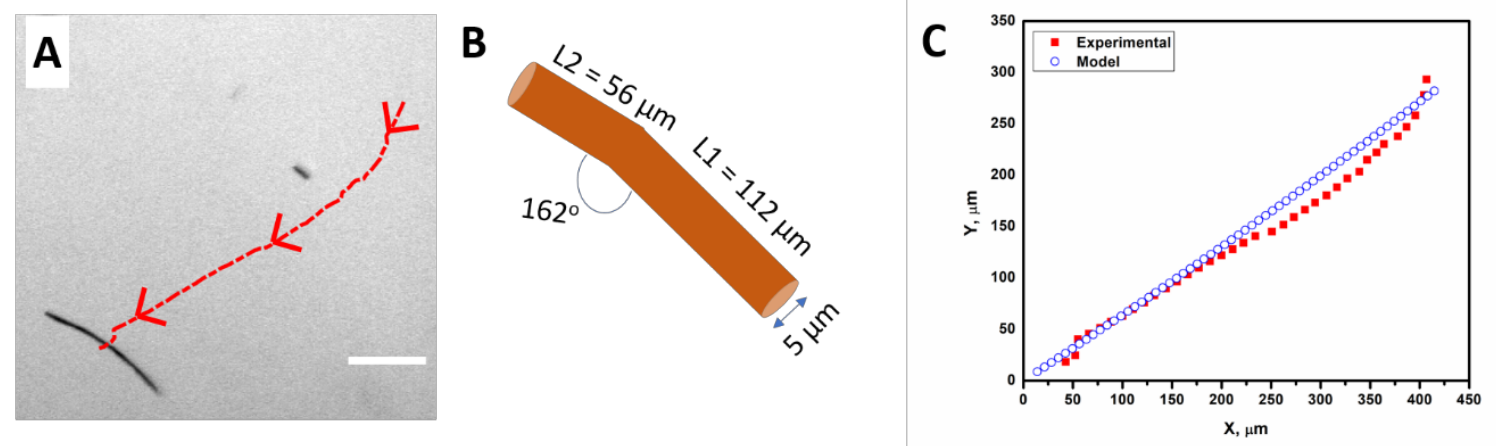

Figure S6. (A) Self-propulsion trajectory of a bent rod in $4 \mathrm{wt} \%$ hydrogen peroxide solution with an average velocity of $13.69 \pm 2.47 \mu \mathrm{m} / \mathrm{s}$. The scale bar is $50 \mu \mathrm{m}$. B) Physical dimensions of the bent rod. C) Predicted and experimental self-propulsion trajectories of the bent rod. The estimated force per unit length is $0.25 * 10^{-13} \mathrm{~N} / \mu \mathrm{m}$. 